\title{
Curcumin/Doxorubicin-encapsulating Nanoparticle IMX-110
}

National Cancer Institute

\section{Source}

National Cancer Institute. Curcumin/Doxorubicin-encapsulating Nanoparticle IMX-110.

NCI Thesaurus. Code C154569.

A water-soluble, nano-sized formulation composed of nanoparticles encapsulating the poorly water-soluble curcumin, a signal transducer and activator of transcription 3 (Stat3), nuclear factor Kappa B (NF-kB) and poly-tyrosine kinase inhibitor (T KI), and the antineoplastic anthracycline antibiotic doxorubicin, with potential antineoplastic activity. Upon administration of the curcumin/doxorubicin-encapsulating nanoparticle IMX-110, the curcumin moiety targets and inhibits the activation of STAT3 and NF-kB and prevents STAT3- and NF-kB-mediated signaling pathways, both of which are activated in a variety of human cancers and plays a key role in neoplastic transformation, uncontrolled tumor cell proliferation, tumor resistance to apoptosis, metastasis and immune evasion. The doxorubicin moiety intercalates into DNA and interferes with topoisomerase II activity. This inhibits DNA replication and RNA synthesis, leading to tumor cell growth inhibition and apoptosis. This agent also interacts with cell membrane lipids causing lipid peroxidation. Delivery of doxorubicin in nanoparticles may improve drug penetration into tumors and curcumin, by inhibiting NFkB and STAT3 activity, may circumvent the tumor cells multidrug resistance mechanisms and may therefore be effective in chemoresistant tumor cells. Chemotherapeutic agents, such as doxorubicin, upregulate the expression of NF-kB in tumor cells which generates chemotherapy-resistant tumor cells. 